\title{
ON ATRIODIC TREE-LIKE CONTINUA ${ }^{1}$
}

\author{
LEX G. OVERSTEEGEN AND E. D. TYMCHATYN
}

\begin{abstract}
D. P. Bellamy has recently shown that atriodic tree-like continua do not have the fixed point property for homeomorphisms. J. B. Fugate and T. B. McLean showed that hereditarily indecomposable tree-like continua have the fixed point property for pointwise periodic homeomorphisms. In this paper the latter result is extended to the case of atriodic tree-like continua. In the course of the proof it is shown that the property of being an atriodic tree-like continuum is a Whitney property. In particular, it is shown that the hyperspace of an atriodic tree-like continuum is at most 2-dimensional.
\end{abstract}

1. Introduction. A continuum is a compact, connected, metric space. A tree is a finite, connected, simply connected, one-dimensional polyhedron. A continuum is tree-like if it admits finite open covers of arbitrarily small mesh whose nerves are trees. A continuum $X$ is said to be a triod (resp. $n$-od) if there exists a subcontinuum $M$ of $X$ such that $X \backslash M$ has at least three (resp. at least $n$ ) components. We say $X$ is atriodic if $X$ contains no triod. A continuum is hereditarily indecomposable if and only if it contains no 2-od.

If $X$ is a continuum we let $C(X)$ denote the hyperspace of subcontinua of $X$ with the Hausdorff metric. A Whitney map for $X$ is a mapping $\mu: C(X) \rightarrow[0, \infty)$ such that $\mu(\{x\})=0$ for each $x \in X$ and $\mu(A)<\mu(B)$ for each $A, B \in C(X)$ with $A \subsetneq B$. A Whitney level for $X$ is a set $\mu^{-1}(t)$ where $0<t<\mu(X)$. Whitney levels are continua in $C(X)$ (see [9, p. 400]). The existence of Whitney maps for $X$ is well known (see [9]).

2. Whitney property. A property $P$ of continua is said to be a Whitney property if whenever $X$ is a continuum with property $\mathrm{P}$ then every Whitney level of $X$ also has property $P$. Krasinkiewicz in [6] and [7] proved that being an arc-like continuum, being a proper circle-like continuum or being an hereditarily indecomposable tree-like continuum is a Whitney property. The main purpose of this section is to show that being an atriodic tree-like continuum is also a Whitney property. This provides a converse to a result of Nadler $[8,3.5]$ who has shown that if $X$ is a continuum whose Whitney levels are tree-like then $X$ is atriodic and tree-like.

A continuum $X$ is said to have the covering property (see [9]) if for each Whitney level $\mu^{-1}(t)$ of $X$ and each subcontinuum $\Lambda$ of $\mu^{-1}(t), \cup \Lambda=X$ implies $\Lambda=\mu^{-1}(t)$.

\footnotetext{
Received by the editors May 27, 1980 and, in revised form, October 31, 1980; presented to the Fourteenth Spring Topology Conference at Birmingham, Alabama, March 1980.

AMS (MOS) subject classifications (1970). Primary 54H25, 54F20, 54B20; Secondary 54F50.

Key words and phrases. Atriodic tree-like continua, pointwise periodic homeomorphism, fixed points, Whitney property.

${ }^{1}$ This research was supported in part by NSERC grant number A5616 and a research grant from the University of Saskatchewan. 
THEOREM 2.1 (SEE [9, p. 485]). A continuum $X$ has the covering property if and only if each Whitney level of $X$ is irreducible.

TheOREm $2.2[4,5.6]$. Atriodic tree-like continua have the covering property.

LEMMA 2.3. Let $X$ be an atriodic tree-like continuum. Let $p \in X$ and let $\mu$ be a Whitney map for $X$. If $\Lambda$ is a subcontinuum of $\mu^{-1}(t)$ for some Whitney level then $K=\Lambda \cap\left\{A \in \mu^{-1}(t) \mid p \in A\right\}$ is an arc or a point or $K$ is empty.

Proof. We suppose $p \in \cup \Lambda$. Since $\cup \Lambda$ is a continuum, it has the covering property by Theorem 2.2. Now $\left.\mu\right|_{C(\cup \Lambda)}$ is a Whitney map for $\cup \Lambda$ and hence $\Lambda$ is a Whitney level of $\cup \Lambda$. By [9, p. 405] $\{A \in \Lambda \mid p \in A\}=K$ is an arcwise connected continuum.

Now $\cup K$ is an atriodic tree-like continuum. By Theorem $2.2 \cup K$ has the covering property. Hence, $K$ is a Whitney level of $\cup K$. By Theorem $2.1 K$ is irreducible.

Sorgenfrey proved in [13, Theorem 1.8] that if $T$ is the union of three continua which have a point in common and such that no one of them is a subset of the union of the other two then $T$ contains a triod.

THEOREM 2.4. The property of being an atriodic tree-like continuum is a Whitney property.

Proof. Let $X$ be an atriodic tree-like continuum and let $\mu^{-1}(t)$ be a Whitney level of $X$. If $\Lambda$ is a subcontinuum of $\mu^{-1}(t)$ then as in the proof of Lemma $2.3 \Lambda$ is a Whitney level of the tree-like continuum $\cup \Lambda$. By [11, Theorem 5] the first Cech cohomology group of $\Lambda$ is trivial.

It follows that $\operatorname{dim} \mu^{-1}(t)=1$. For if $Y$ is a continuum with $\operatorname{dim} Y>2$ then there exists an essential map $f$ of $Y$ onto $B$, the closed unit disk in the plane [10, p. 127]. Then $\left.f\right|_{f^{-1}\left(S^{1}\right)}$ is essential where $S^{1}$ is the boundary of $B$ and, hence, $f$ is essential on some subcontinuum $Z$ of $f^{-1}\left(S^{1}\right)$. i.e. $H^{1}(Z) \neq 0$.

Let $K=\left\{(A, x) \mid x \in A \in \mu^{-1}(t)\right\} \subset \mu^{-1}(t) \times X$. Then $K$ is a continuum and $\operatorname{dim} K \leqslant 2$. Let $\pi_{1}: K \rightarrow \mu^{-1}(t)$ and $\pi_{2}: K \rightarrow X$ be the coordinate projections. The point inverses under $\pi_{1}$ are tree-like continua and the point inverses under $\pi_{2}$ are arcs or points by Lemma 2.3. In particular, the point inverses of $\pi_{1}$ and $\pi_{2}$ have trivial shape. By a theorem of Sher [12] $\mu^{-1}(t)$ and $X$ have the same shape since $\pi_{1}$ and $\pi_{2}$ are cell-like mappings between finite dimensional spaces. By the theorem of Case and Chamberlin [2] $\mu^{-1}(t)$ is tree-like.

By Theorem $2.2 X$ has the covering property hereditarily. Therefore, by $[9, \mathrm{p}$. $510] \mu^{-1}(t)$ is hereditarily irreducible and, hence, $\mu^{-1}(t)$ is atriodic. This completes the proof of the theorem.

COROLlary 2.5. If $X$ is an atriodic tree-like continuum, then $C(X)$ is 2-dimensional.

3. The fixed point theorem. In [3] Fugate and McLean proved the following two results.

THEOREM 3.1 [3, 1.5]. Tree-like continua have the fixed point property for periodic homeomorphisms. 
TheOREM $3.2[3,3.3]$. Hereditarily indecomposable tree-like continua have the fixed point property for pointwise periodic homeomorphisms.

In this section we extend Theorem 3.2 to the case of atriodic tree-like continua. In our argument we use Theorem 2.4 and follow the argument given in [3]. First we prove the following lemma.

LEMMA 3.3. If $M$ is an atriodic, hereditarily unicoherent continuum and if $h$ : $M \rightarrow M$ is a pointwise periodic homeomorphism, then the induced homeomorphism $\bar{h}$ : $C(M) \rightarrow C(M)$ which is defined by $\bar{h}(Y)=h(Y)$ for each $Y \in C(M)$ is pointwise periodic. Moreover, if $x \in A \in C(M)$ and $h^{n}(x)=x$, then $\bar{h}^{2 n}(A)=A$.

Proof. Let $x \in A \in C(M)$ and suppose $h^{n}(x)=x$. If $y \in A$ then there is a unique continuum $B_{y}$ in $M$ which is irreducible from $x$ to $y$ since $M$ is hereditarily unicoherent. Then $B_{y} \subset A$ and $A=\cup\left\{B_{y} \mid y \in A\right\}$. Let $y \in A \backslash\{x\}$ and let $B=B_{y}$. It suffices to show that $h^{2 n}(B)=B$. Suppose $h^{2 n}(B) \neq B$. If $B \subsetneq h^{n}(B)$ let $z \in h^{n}(B) \backslash B$. Then $h^{i n}(z) \in h^{(i+1) n}(B) \backslash h^{i n}(B)$ for each $i$. Since $h^{i n}(B) \subset$ $h^{(i+1) n}(B)$ for each positive integer $i$ this would imply $z$ has infinite order under $h$ and so would contradict the pointwise periodicity of $h$. Thus, $B \not \subset h^{n}(B)$. Similarly, $h^{n}(B) \not \subset B$. Notice that $h^{n}(y) \notin B$ since $B \cap h^{n}(B)$ is a proper subcontinuum of $h^{n}(B)$ and $h^{n}(B)$ is irreducible between $h^{n}(x)=x$ and $h^{n}(y)$. Similarly, $h^{n}(y) \notin$ $h^{2 n}(B), h^{2 n}(y) \notin B \cup h^{n}(B)$ and $y \notin h^{n}(B) \cup h^{2 n}(B)$. By Sorgenfrey's theorem [13, 1.8] $M$ contains a triod. This is a contradiction. Thus, we have proved $B=h^{2 n}(B)$ and, hence, $A=\bar{h}^{2 n}(A)$.

THEOREM 3.4. Suppose $M$ is an atriodic tree-like continuum and $h: M \rightarrow M$ is a pointwise periodic homeomorphism. Then $h$ has a fixed point.

Proof. Suppose $h$ does not have a fixed point. We may suppose $M$ is minimal with respect to being mapped into itself. Hence, $M$ is not a point and if $Y$ is a proper subcontinuum of $M, h(Y) \not \subset Y$.

Let $\mu$ be a Whitney map for $M$. We may suppose $\mu(M)=1$. Let $\bar{h}: C(M) \rightarrow$ $C(M)$ be the map induced by $h$.

For $x \in M$ let $O(x)=\min _{n>0}\left(h^{n}(x)=x\right)$. Let $J_{i}=\{x \in M \mid O(x)<i\}$. Then $J_{i}$ is closed and $M=J_{1} \cup J_{2} \cup \ldots$ By the Baire Category Theorem there exists $n$ such that $J_{n}$ has nonvoid interior in $M$. From the above it follows that there exists $s$ with $0<s<1$ such that if $K \in \mu^{-1}([s, 1])$ then $K \cap J_{n} \neq \varnothing$.

Define $\sigma: C(M) \rightarrow[0,1]$ by

$$
\sigma(A)=\max \left\{\mu\left(\bar{h}^{i}(A)\right) \mid 1 \leqslant i \leqslant 2 n !\right\} .
$$

Then $\sigma$ is clearly a Whitney map for $M$ such that if $\mu(A) \geqslant s$ then $\sigma(\bar{h}(A))=\sigma(A)$. Since $\mu^{-1}(1)=\sigma^{-1}(1)=\{M\}$ there exists $0<t<1$ such that $\sigma(A) \geqslant t$ implies $\mu(A) \geqslant s$.

By Theorem $2.4 \sigma^{-1}(t)$ is a tree-like continuum in $C(M)$. The restriction of $\bar{h}$ to $\sigma^{-1}(t)$ is a periodic homeomorphism of $\sigma^{-1}(t)$ (of period $<2 n$ !). By Theorem 3.1 $\bar{h}(A)=A$ for some $A \in \sigma^{-1}(t)$. This contradicts the assumption at the beginning of the proof that $\bar{h}(Y) \not Y$ for each proper subcontinuum $Y$ of $M$. 
Question. Is Theorem 3.4 true for tree-like continua which do not contain $n$-ods for arbitrarily large $n$ ?

\section{REFERENCES}

1. D. P. Bellamy, A tree-like continuum without the fixed point property, Houston J. Math. 6 (1980), 1-13.

2. J. H. Case and R. E. Chamberlin, Characterizations of tree-like continua, Pacific J. Math. 10 (1960), 73-84.

3. J. B. Fugate and T. B. McLean, Compact groups of homeomorphisms on tree-like continua (to appear).

4. J. Grispolakis and E. D. Tymchatyn, Continua which are images of weakly confluent mappings only. I, Houston J. Math. 5 (1979), 483-502.

5. __ On confluent mappings and essential mappings, Rocky Mountain J. Math. (to appear).

6. J. Krasinkiewicz, On the hyperspaces of hereditarily indecomposable contimua, Fund. Math. 84 (1974), 175-186.

7.

8. S. B. Nadler, Jr., Whitney-reversible properties, Fund. Math. (to appear).

9. __ Hyperspaces of sets, Dekker, New York, 1978.

10. A. R. Pears, Dimension theory of general spaces, Cambridge Univ. Press, Cambridge, 1975.

11. J. T. Rogers, Jr., Applications of a Vietoris-Begle theorem for multi-valued maps to the cohomology of hyperspaces, Michigan Math. J. 22 (1976), 315-319.

12. R. B. Sher, Realizing cell-like maps in Euclidean space, General Topology Appl. 2 (1972), 75-89.

13. R. H. Sorgenfrey, Concerning triodic continua, Amer. J. Math. 66 (1944), 439-460.

Department of Mathematics, University of Alabama in Birmingham, Birmingham, Alabama 35294

Department of Mathematics, University of Saskatchewan, Saskatoon, Saskatchewan, CANADA S7N OWO 